
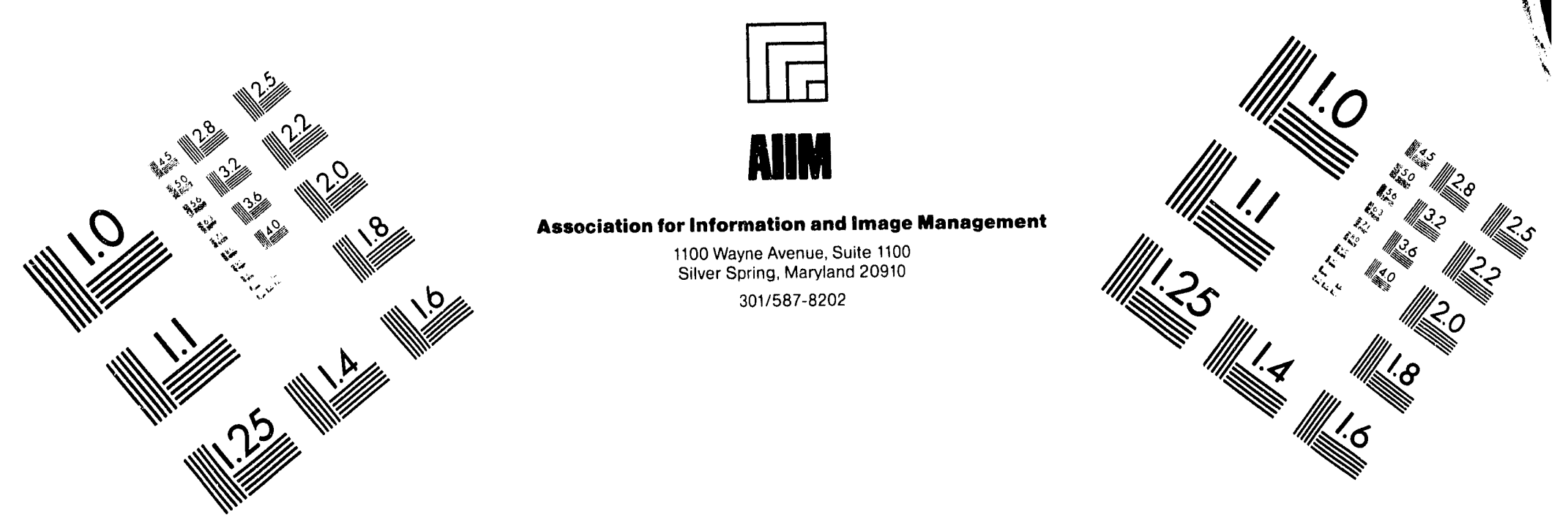

\title{
Centimeter
}

$\begin{array}{llllllllllllllll}1 & 2 & 3 & 4 & 5 & 6 & 7 & 8 & 9 & 10 & 11 & 12 & 13 & 14 & 15 & \mathrm{~mm}\end{array}$

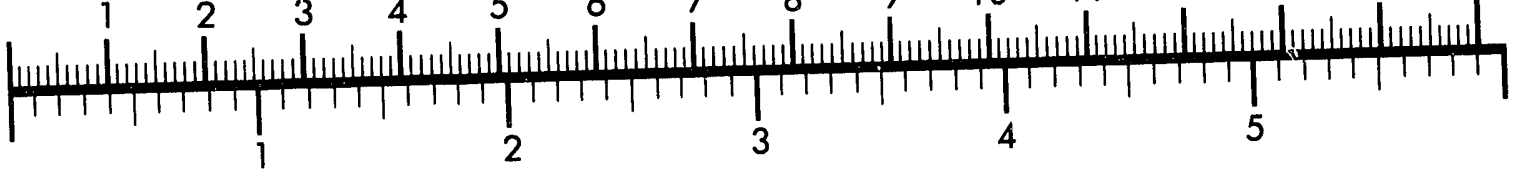
Inches
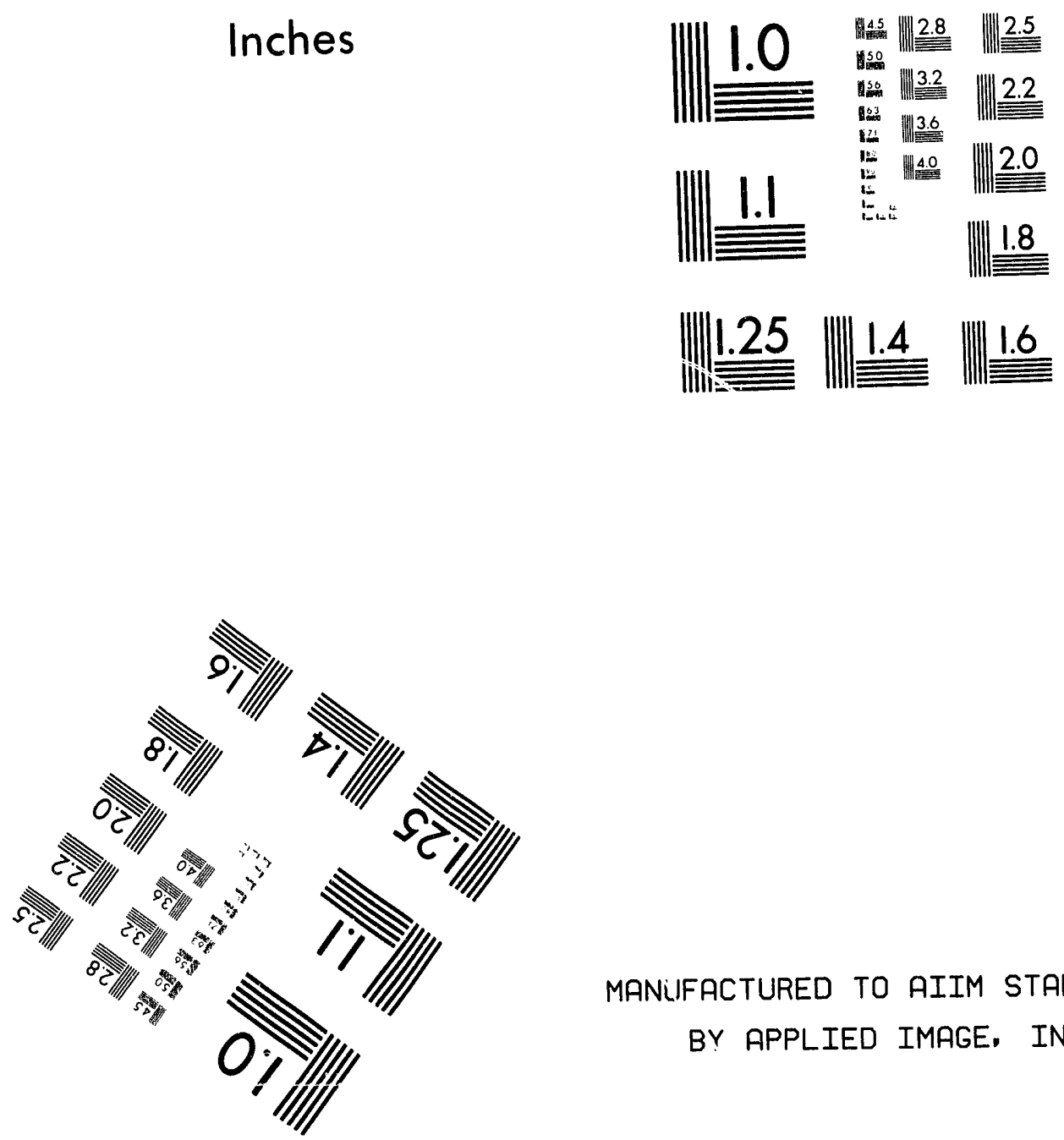

MANUIFACTURED TO AIIM STANDARDS BY APPLIED IMAGE, INC.

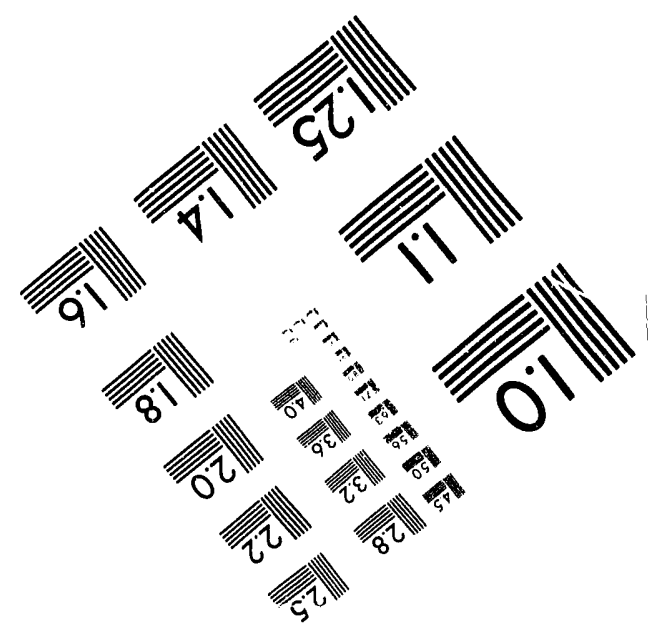



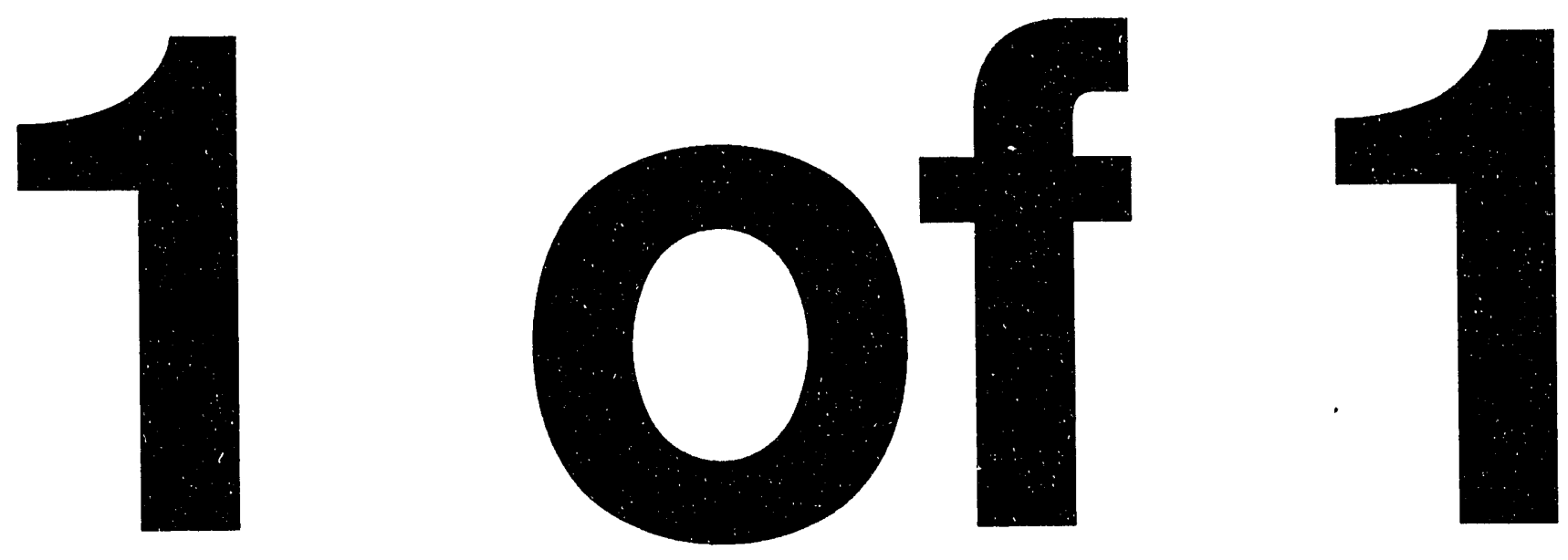


\section{Overall Risk Estimation for Nonreactor Nuclear Facilities and Implementation of Safety Goals $(U)$}

K. S. Kim

R. F. Bradley

Publication Date: June 1993

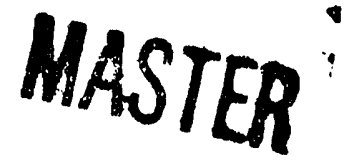

Westinghouse Savannah River Company Savannah River Site

Aiken, SC 29808

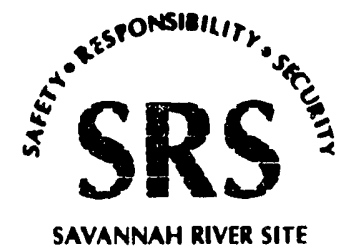

Prepared for the U. S. Department of Energy under Contract No. DE-AC09-89SR18035 
BLANK 


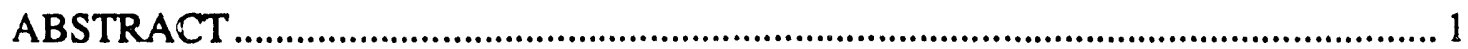

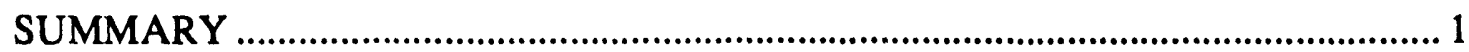

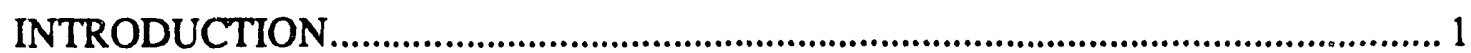

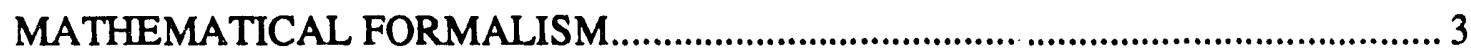

General Formalism and Expected Value.....................................................................................................

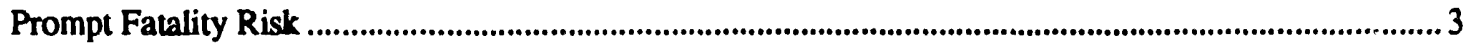

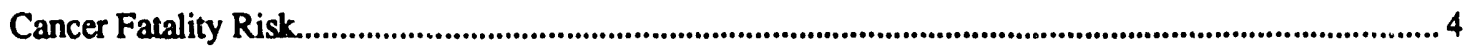

PROTOCOL OF ESTABLISHING EXCEEDANCE PROBABLITY ......................... 5

Release Fraction.................................................................................................................................. 5

Individual Radiation Dose.................................................................................................................................6

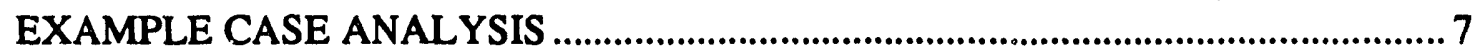

Baseline Data from SAR and Dose Adjustment ..........................................................................................

Confinement Capability and Source Terms......................................................................................................... 8

Prompt Fatality............................................................................................................................................................... 8

Cancer Fatality...................................................................................................................................................... 8

EVALUATION OF RISK COMPARISON GUIDELINE ............................................... 9

CONCLUDING REMARKS ...................................................................................

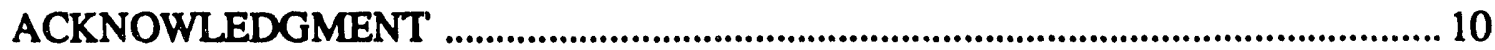

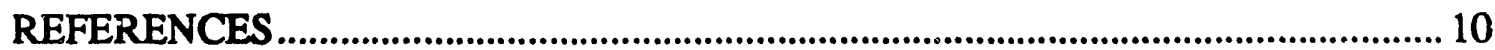

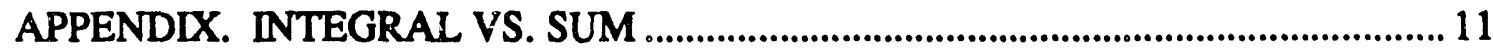




\section{LIST OF TABLES}

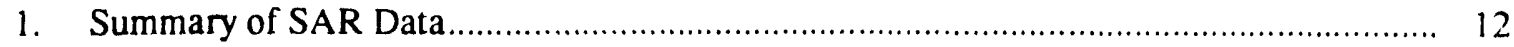

2. Summary of Adjusted Dose Data ......................................................................... 13

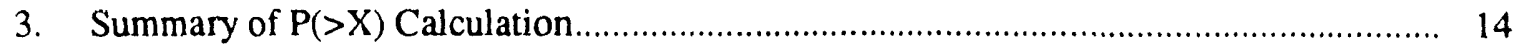

4. Summary of $\mathrm{P}(>\mathrm{D})$ Calculation (Site Boundary Acute Dose for Prompt Fatality)........... 14

5. Summary of $\mathrm{P}(>\mathrm{D})$ Calculation (Average CEDE within 10 miles for Cancer Fatality) ..... 15

\section{LIST OF FIGURES}

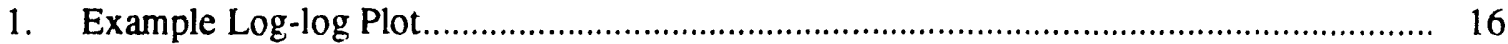

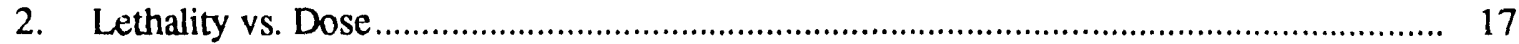

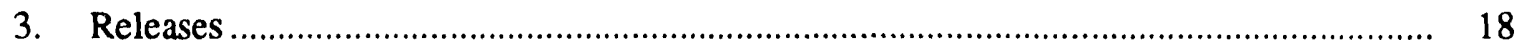

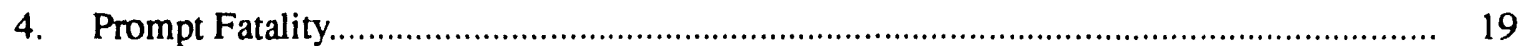

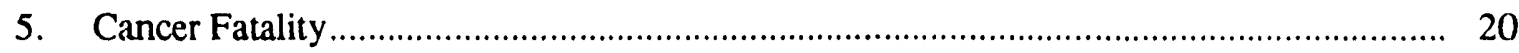

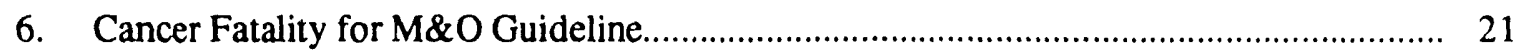




\title{
OVERALL RISK ESTIMATION FOR NONREACTOR NUCLEAR FACILITIES AND IMPLEMENTATION OF SAFETY GOALS
}

\author{
by \\ Kyo S. Kim and R. Foster Bradley \\ Westinghouse Savannah River Company \\ Savannah River Site \\ Aiken, SC 29808
}

\begin{abstract}
A typical safety analysis report (SAR) contains estimated frequencies and consequences of various design basis accidents (DBA). However, the results are organized and presented in such a way that they are not conducive for summing up with mathematical rigor to express total or overall risk. This paper describes a mathematical formalism for deriving total risk indicators. The mathematical formalism is based on the complementary cumulative distribution function (CCDF) or exceedance probability of radioactivity release fraction and individual radiation dose. A simple protocol is presented for establishing exceedance probabilities from the results of DBA analyses typically available from an SAR. The exceedance probability of release fraction can be a useful indicator for gaining insights into the capability of confinement barriers, characteristics of source terms, and scope of the SAR. Fatality risks comparable to the DOE Safety Goals can be derived from the exceedance probability of individual doses. Example case analyses are presented to illustrate the use of the proposed protocol and mathematical formalism. The methodology is finally applied to proposed risk guidelines for individual accident events to show that these guidelines would be within the DOE Safety Goals.
\end{abstract}

\section{SUMMARY}

Expressing the results of Safety Analysis Reports (SAR) in terms of the complementary cumulative distribution function (exceedance probability) is extremely useful. For a specific facility, the exceedance probability of the release fraction provides insight into the charcteristics of source terms and confinement capabilities of the engineered barrier systems. The exceedance probability of individual dose provides a means to determine overall risk for a facility. Exceedance probability of release fraction also provides an effective measure of the completeness of scenarios included in a particular facility SAR.

The exceedance probability of individual dose can be applied on a sitewide basis to provide a determination of risk to individuals in terms of fatality risks. The exceedance probability method is more mathematically rigorous and conservative in determining overall risk than simply summing up individual accident risks.

The Westinghouse $\mathrm{M} \& O$ guidelines for radiological risk compurison is a continuous curve on a Farmer plot for evaluating the acceptability of consequences at the site boundary of individual accident scenarios. When this guideline is treated as the exceedance probability of individual dose, it is found to be well within the DOE Nuclear Safety Policy Goals for prompt fatality for the Savanniah River Site. It is also within the goal for latent cancer fatality risk by a factor of approximately 15 .

\section{INTRODUCTION}

A nuclear facility Safety Analysis Report (SAR) written according to Department of Energy (DOE) or Nuclear Regulatory Commission (NRC) guidelines ${ }^{1,2}$ is primarily a design oriented document. It contains analyses of Design Basis Accidents (DBA), which are "deterministic" in principle. The results of DBA analyses are presented in terms of radiological exposure to an individual and to the population. However, the primary purpose of the analyses is to establish and justify the design parameters of various safety systems and to demonstrate regulatory compliance, rather than to quantify the radiological risk to the general population in the vicinity of a nuclear facility or site. 
Another characteristic of the SAR-DBA analysis is that it is a "piecemeal" analysis. That is, a DBA analysis deals with one accident scenario at a time and what is presented in a typical SAR is a list of postulated accidents with associated consequences and the event frequencies. The frequencies and consequences of various accident sequences are generally organized and presented in such a way that they are not conducive for "summing up" with mathematical rigor to express "total or overall risk". This is a lesser issue for commercial reactors safety analyses, since a large scale core-melt accident is a good surrogate for total risk and core melt frequency and associated prompt and cancer fatalities can provide the "total" risk picture. However, for a typical nonreactor nuclear facility, there is not always a single dominant accident sequence that can be a reasonable surrogate for the total risk. Instead, there may be several accident scenarios that can significantly contribute to the total risk.

The need for estimation of total risk has become more apparent due to a recent DOE publication of Nuclear Safety Policy ${ }^{3}$ containing quantitative Safety Goals as summarized below;

The risk to an average individual in the vicinity of a DOE nuclear facility for prompt fatalities that might results from accidents should not exceed $0.1 \%$ of the sum of prompt fatalities resulting from other accidents to which members of the population are generally exposed. For evaluation purposes, individuals are assumed to be located within one mile of the site boundary.

The risk to the population in the area of a DOE nuclear facility for cancer fatalities that might result from operations should not exceed $0.1 \%$ of the sum of all cancer fatality risks resulting from all other causes. For evaluation purposes, individuals are assumed to be located within 10 miles of the site boundary.

The following observations can be made from the DOE Safety Goals;

1. They are identical to the NRC Safety Goals for commercial nuclear reactors minus the core-melt frequency goal.
2. Typical SARs do not contain risk indicators (i.e., fatality risk) readily comparable to the quantitative Safety Goals.

3. Some type of probabilistic risk assessment (PRA) including consequence analysis is necessary to obtain results comparable to the quantitative Safety Goals.

4. A risk indicator comparable to the Safety Goals should represent the total accident risk of a facility (not just worst accident risk). For a multifacility site, the risk indicator should represent all facilities within the site.

In view of total number of DOE facilities/sites, type of facilities, and nature and degree of risk, a Level 3 PRA for all DOE facilities may be impractical or unjustifiable. This paper describes a mathematical formalism of deriving total risk indicators. The mathematical formalism is based on the complementary cumulative distribution function (CCDF) or simply called "exceedance probability" of radioactivity release fraction and individual radiation dose. A simple protocol is presented for establishing exceedance probabilities from SAR-DBA analyses. The protocol makes maximum use of the results of DBA analyses typically available from a SAR. The exceedance probability of release fraction, denoted by $\mathrm{P}(>\mathrm{X})$ can provide insight into the capability of confinement barriers and characteristics of source terms. Prompt and cancer fatality risks comparable to the quantitative Safety Goals can be derived from the exceedance probability of individual dose denoted by $\mathrm{P}(>\mathrm{D})$.

The following sections describe a general mathematical formalism involving exceedance probability and mathematical derivations of total prompt and cancer fatalities from exceedance probabilities followed by a protocol for establishing exceedance probabilities from SAR data. An Example Case Analysis is presented to illustrate how the protocol and mathematical formalism work and how they can be applied to SARs.

Finally, the methodology is applied to the recently proposed Westinghouse $\mathrm{M} \& \mathrm{O}$ risk guidelines ${ }^{4}$ to show the validity of these guidelines with respect to the DOE Safety Goals. 


\section{MATHEMATICAL FORMALISM}

The following mathematical formalism is an extension of that presented by Munera-Yadigaroglu ${ }^{5}$ and Cox-Baybutt. ${ }^{6}$

\section{General Formalism and Expected Value}

In the following formalism, the exceedance probability, $\mathrm{P}(>\mathrm{C})$ is defined as "annual probability of events whose consequences exceed $\mathrm{C}^{\prime}$. By defining the exceedance probability on an "annual" basis, this paper adopts both "objectivist (or frequentist)" and "subjectivist" viewpoints of the meaning of the probability. ${ }^{8}$ Hence, the terms "annual probability" and "annual frequency" are interchangeably used throughout this paper. Some minor SAR data adjustment (i.e., those events more frequent than once a year) is needed, which is discussed in the next section. Assuming that the probability distribution of consequence is a continuous function of consequence, the above definition can be expressed by the following equations:

$P(>C)=\int_{C}^{C_{\max }} g\left(C^{\prime}\right) \cdot d C^{\prime}$

and $d P(>C)=-g(C) \cdot d C$

where $g(C)$ is a probability (or frequency) distribution function (PDF) of consequences. Using the above definitions and integration by parts, the integral of $\mathrm{P}(>\mathrm{C})$ over the entire consequence range can be expressed:

$$
\begin{aligned}
\int_{0}^{C_{\max }} P(>C) \cdot d C & =[C \cdot P(>C)]_{0}^{C \max } \cdot \int_{0}^{C_{\max }} C \cdot d P(>C) \\
& =0+\int_{0}^{C_{\max }} C \cdot g(C) \cdot d C
\end{aligned}
$$

The last term of the above equation is an "expected value of $\mathrm{C}^{n}$ (i.e., risk) by definition. Hence,

$\int_{0}^{C_{\max }} P(>C) \cdot d C=C_{\text {exp. }}$ (expected value of $\left.C\right)$
It should be noted that the consequence, $\mathrm{C}$ can bc either release of radioactivity or individual radiation dose and the resulting integration of exceedance probability represents the expected annual release (curies/yr) or expected annual individual dose (rem/yr) covering all accidents. It should also be noted that the units of the above indicators (i.e., curies/yr or rem/yr) are the same as those of "risk" when the risk is defined by probability (or frequency) times consequence.* In some SARs, a "total risk" is calculated by a summation of individual "risk" of various scenarios. Since a typical SAR includes only selected discrete accident scenarios, such a summation may be an underestimation. A comparison between integration of exceedance probability and summation of individual events is shown in the Appendix.

\section{Prompt Fatality Risk}

As mentioned previously, the exceedance probability of individual dose, $\mathrm{P}(>\mathrm{D})$ can be used to derive overall fatality risk for a facility or a site. As in the case of $\mathrm{P}(>\mathrm{C}), \mathrm{P}(>\mathrm{D})$ is defined:

$P(>D)=\int_{D}^{D_{\max }} g\left(D^{\prime}\right) \cdot d D^{\prime}$

and $d P(>D)=-g(D) \cdot d D$

where $g(D)$ is a PDF of individual dose

The risk of an individual promptly dying from acute radiation exposure (denoted by $F_{p}$ ) due to all accidents (all dose ranges) can be expressed as:

$F_{p}=\int_{0}^{D_{\max }} q(D) \cdot g(D) \cdot d D$

where $q(D)$ is the probability of prompt fatality due to receiving acute dose of $D$ rem (dose response function).

\footnotetext{
* As pointed out by many authors (for example, see Reference 8 ), representation of risk by a single value indicator such as probability times consequence may be meaningless and misleading. A better representation of risk is probability and consequence.
} 
The exact functional form of $q(D)$ is not well established. The simplest case would be assuming that $q(D)$ is a step function, that is :

$q(D)=0$ if $D<D_{L}$, and

$q(D)=1$ if $D \geq D_{L}$

where $D_{L}$ represent $L_{50 / 60}$ (50\% of dying in 60 days) or a similar criterion. Then Eq (6) becomes:

$$
\begin{aligned}
F_{p} & \left.=\int_{0}^{D_{L}} q(D) \cdot g(D) \cdot d D=\int_{D_{L}}^{D_{\max }} q(D) \cdot g(D) \cdot d D\right) \\
& =0+\int_{D_{L}}^{D_{\max }} g(D) \cdot d D
\end{aligned}
$$

By definition of Eq (4)

$F_{p}=P\left(>D_{L}\right)$

In other words, in the case of a step function dose response, the prompt fatality risk is simply the annual probability of a person receiving greater than $D_{L}$ rem.

The dose-lethality relationship for a "normal man" in the literature ${ }^{7}$ is plotted in Figure 2. As can be seen in this figure, a linear dose response is approximated within a range $\left(D_{1}, D_{2}\right)$, that is;

$q(D)=0$ for $D<D_{1}$

$q^{\prime}(D)=1 /\left(D_{2}-D_{1}\right)$ for $D_{1} \leq D<D_{2}$

$q(D)=1$ for $D \geq D_{2}$,

where $q^{\prime}(D)$ is $d q(D) / d D$.

Subsequently, Eq (6) can be rewritten as:

$F_{p}=\int_{0}^{D_{1}} q(D) \cdot g(D) \cdot d D+$

$\int_{D 1}^{D_{2}} q(D) \cdot g(D) \cdot d D+\int_{D_{2}}^{D_{\max }} q(D) \cdot g(D) \cdot d D$

The first term becomes 0 , since $q(D)=0$ in the domain $\left(0, D_{1}\right)$. The third term becomes $P\left(>D_{2}\right)$ by definition, since $q(D)=1$. Due to $E q(5)$ and integration by parts, the second term becomes:

$$
\begin{aligned}
&-\int_{D_{1}}^{D_{2}} q(D) \cdot d P(>D)= \\
& {\left.[-q(D) \cdot P(>D)]_{D_{1}}^{D_{2}}+\int_{D_{1}}^{D_{2}} q^{\prime}(D) \cdot P>D\right) \cdot d D } \\
& \\
&=-P\left(>D_{2}\right)+\left(\frac{1}{D_{2}-D_{1}}\right) \cdot \int_{D_{1}}^{D_{2}} P(>D) \cdot d D
\end{aligned}
$$

Hence,

$F p=\left(\frac{1}{D_{2} \cdot D_{1}}\right) \cdot \int_{D_{1}}^{D_{2}} P(>D) \cdot d D$

It should be emphasized that the radiation dose $D$ in the above prompt fatality derivation is an acute dose, not a 50-year cumulative dose.

\section{Cancer Fatality Risk}

The risk of latent cancer fatality (denoted by $F_{c}$ ) can be calculated in a similar fashion. The mathematical formalism is the same as the case of Prompt Fatality. It should be noted that $P(>D)$ in this calculation is based on average individual dose (Committed Effective Dose Equivalent or CEDE) within 10 miles from the site boundary .

$F_{c}=\int_{0}^{D_{\max }} c(D) \cdot g(D) \cdot d D$

where $c(D)$ is probability of developing fatal cancer due to receiving $D$ rems (CEDE). The relationship between cancer incident rate vs. low dose is a controversial subject, and there are no consensus mathematical expressions of this relationship. For regulatory purposes, the Federal regulatory agencies (e.g., NRC, EPA) adopted the "no threshold linear theory", which means that $c(D)$ is a linear function of $D$, hence:

$c^{\prime}(D)=d c / d D=K$ (cancer rate per rem).

Using the same steps for deriving $\mathrm{Eq}$ (11), the Eq (12) becomes

$$
\begin{aligned}
F_{c} & =K \cdot \int_{0}^{D_{\max }} P(>D) \cdot d D \\
& =K \cdot D_{\exp }
\end{aligned}
$$


In summary, the total fatality risk can be estimated from the exceedance probabilities of individual dose, $P(>D)$ for an average person at the site boundary (Prompt Fatality) and within 10 miles of site boundary (Cancer Fatality). It is interesting to note that PDF does not appear explicitly in the final expressions of fatality risk formalism (i.e., equations
8,11 , and 13 ), only exceedance probability does. This means that the total fatality risk can be calculated without the knowledge of exact PDF, if one can establish the exceedance probabilities by some other means. The following section discusses how to establish the exceedance probabilities from the results of SAR-DBA analyses.

\section{PROTOCOL OF ESTABLISHING EXCEEDANCE PROBABILITY}

In the above mathematical formalism, a continuous PDF was assumed for defining the exceedance probability (CCDF). However, a typical SAR does not provide a continuous PDF, but it provides discrete probabilities of accident sequences (Farmer plot). Ever since the publication of Farmer's original proposal regarding use of "Limit Line" for risk acceptability decisions, ${ }^{9}$ there were controversies and confusion regarding the meaning of the Limit Line. It is now generally agreed ${ }^{5}$ that the Farmer's Limit Line is indeed a form of PDF (logarithmic), but Cox and Baybutt suggested ${ }^{6}$ that a Limit Line based on CCDF is a better tool to assess overall risk. In the discussion of the Discrete Model, Cox and Baybut defined the cumulative distribution function of consequences by:

$$
\begin{aligned}
F(C) & =\text { Prob (consequences less than or equal to } C \text { ) } \\
& =\sum_{C_{i} \geq C} f_{i}
\end{aligned}
$$

and

$$
\text { Complementary } F(C)=\sum_{C_{i}>C}^{C_{\max }} f_{i} \text {, with a boundary }
$$

condition

$\sum \mathrm{fi}_{\mathrm{i}}=1.0$,

All $\mathrm{Ci}_{\mathrm{i}}$

where the sequence fi's is called the probability function of respective consequences $\mathrm{Ci}$. It should be noted that the Complementary $F(C)$ is the same as
$\mathrm{P}(>\mathrm{C})$ in this paper. The procedure for establishing $F(C)$ and complementary $F(C)$ is also shown graphically in the Appendix of Reference 6. Kaplan and Garrick presented a similar protocol. ${ }^{8}$ The protocol presented below is according to these references which is in essence a successive summation of individual event frequencies (or annual probabilities) according to consequence groupings. This protocol is valid if the Limit Line is dominated by a few accident scenarios and the density of points (i.e., accident sequences) near the Limit Line is small. ${ }^{10}$ The exceedance probability protocols discussed below for radioactivity release fraction and individual radiation dose are the same.

\section{Release Fraction}

The exceedance probability of release fraction, $P(>X)$ where $X$ represents the radioactivity release fraction, is defined as an annualized probability of a discrete release exceeding $X$. The protocol is as follows:

1. Search all radioactivity release and radiation dose estimates from an SAR for all postulated events with associated annual frequencies or probabilities.

2. Evaluate each scenario/event to assure that selected events are independent from each other (events are mutually exclusive), and make necessary adjustment on the frequency and

\begin{tabular}{|c|c|c|c|c|c|}
\hline Event\# & Description & $\begin{array}{l}\text { Frequency } \\
\text { (/yr) }\end{array}$ & $\begin{array}{l}\text { Release } \\
\text { (Curie) }\end{array}$ & $\begin{array}{l}\text { Release } \\
\text { (Fraction) }\end{array}$ & Dose(rem) \\
\hline 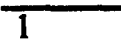 & Fire in XYZ & $f_{1}$ & $\overline{R_{1}}$ & $\mathrm{X}_{1}$ & $\mathrm{D}_{1}$ \\
\hline 2 & Chem React & $f_{2}$ & $\mathbf{R}_{2}$ & $x_{2}$ & $\mathrm{D}_{2}$ \\
\hline - & - & - & _- & - & - \\
\hline $\mathrm{i}$ & $\mathrm{ABC}$ & $\mathbf{f}_{\mathbf{i}}$ & $\mathbf{R}_{\mathbf{i}}$ & $\mathrm{Xi}$ & $\mathrm{D}_{\mathrm{i}}$ \\
\hline
\end{tabular}
consequence estimation as needed. Organize the results in a tabular form as shown in the following example: 
3. The release fraction, $X_{\mathrm{i}}$ is calculated by dividing $R_{i}$ by the total curie inventory in the facility.

4. Reorganize the events according to release fraction starting from the highest possible $X_{i}$ $(X=1.0$ for example). The interval of release category can be chosen based on data structure established in step 2.

5. Calculate $P(>X)$ by successively adding annual frequency, $f_{i}$.

6. Stop when $\mathrm{P}(>\mathrm{X})$ reaches 1.0 . By limiting $\mathrm{P}(>\mathrm{X})$ less than 1.0, we are lumping all those events more frequent than once a year into one release category (i.e., normal operation) as well as satisfying the boundary condition. The release value (or release fraction) in such case should be adjusted (see Footnote 4 of Table 1).

7. Summarize the results in a tabular form as shown in the following example. Plot the $P(>X)$ 's vs. $X$ on log-log paper as shown in Figure 1. Establish a Limit Line by connecting highest $P(>X)$ points. The points falling below the Limit Line (such as points "A" and "B" in Figure 1) can be real (i.e., real characteristics of confinement design), but are more likely due to un-analyzed events in the SAR.

\section{Release}

$\begin{array}{lll}\text { Eraction }(\mathbf{X}) & \text { Event } \# & \mathbf{P}(2 \mathbf{X}) .(/ \mathrm{yr}) \\ 1.0 & 0 & \mathrm{f}_{0} \\ 1.0(-1) & 0+1 & \mathrm{f}_{0}+\mathrm{f}_{1} \\ 1.0(-2) & 0+1+2+3 & \mathrm{f}_{0}+\mathrm{f}_{1}+\mathrm{f}_{2}+\mathrm{f}_{3} \\ - & - & - \\ - & - & 1.0\end{array}$

8. A catastrophic event frequency $\left(f_{0}\right)$ releasing the total inventory $(X=1.0)$ is not generally estimated in SARs. This is more likely when the analyzed events do not cover the frequency range of $10^{-6}$ to $10^{.7}$ per year, which is the range of the fatality Safety Goal. In such a case, $f_{0}$ may have to be estimated by extrapolating adjacent data points.

A $\log -\log$ plot of exceedance probability of radioactivity fraction is a useful tool to gain insights into the confinement performance and source term characteristics. Since the plot is based on release fraction instead of absolute amount of radioactivity, it provides a graphic indication of how comprehensive an SAR is in terms of covering the entire release spectrum (i.e., from normal release to $100 \%$ release). Further discussion regarding confinement performance and $P(>X)$ plot is presented in the next section of an Example Case Analysis.
It should be emphasized that $P(>X)$ is an indicator for an individual facility (or even a segment of a facility depending on source terms). More than one $P(>X)$ may be established for a facility, if the facility contains very different species of radioactivity in terms of release characteristics. For example, $P(>X)$ for gaseous species and for particulate may be separately defined to get a more detailed understanding of confinement performance.

\section{Individual Radiation Dose}

The protocol of establishing $P(>D)$ based on SAR dose estimation is the same as the protocol of $P(>X)$. That is, list all events with annual frequencies and dose consequences. Reorder the events according to dose category from maximum dose to minimum dose, and sum up all annual frequencies within the category and categories before. Present the result as a table and $\log -\log$ plot the same way as $\mathrm{P}(>\mathrm{X})$.

It should be noted that the accident radiation dose values presented in a SAR are generally 50-year cumulative dose. It should further be noted that shortterm external or "shine" contributions are typically neglected in the SAR, since they are much smaller than 50-year cumulative dose (committed). Therefore, some adjustments need to be made to SAR dose values for establishing $P(>D)$ for estimation of the prompt fatality risk, since the prompt fatality formula is based on acute dose response function. For example, $L_{50 / 60}$ values depicted in Figure 2 are based on the short term external whole body dose. Conversion of cumulative dose (or CEDE) to acute whole body dose with a simple modification factor may be possible depending on the type of radiation involved in the accident sequences. The latent cancer fatality is based on cumulative dose response function and the above modification is not necessary. A simple modification case is discussed with an Example Case Analysis in the next section together with a modification due to atmospheric dispersion factors (x/Q).

For a multifacility site, total site $P(>D)$ can be obtained by graphically adding the Limit Lines of each facility. Since the events and frequencies are selected in such a way that they are mutually exclusive, it is possible to add Limit Lines to obtain total exceedance probability. For example, the total fatality risk in WASH $1400^{11}$ is obtained by adding Limit Lines of various accidents (e.g., flood, mine accidents, airplane crash, etc.). 


\section{EXAMPLE CASE ANALYSIS}

This section presents an example case analysis of three nonreactor nuclear facilities located at the DOE's Savannah River Site (SRS). Facility \#1 processes radioactive liquid/solution containing fission products (predominantly Cs-137) by solidification, Facility \#2 processes radioactive solids containing $\mathrm{Pu}-238$ by hot pressing, and Facility \#3 processes radioactive gas ( $\mathrm{H}-3)$ by purification and bottling. It should be emphasized that the following example case analysis is only to illustrate the protocol and mathematical formalism. Many assumptions used in the analysis may be overly simplistic, and need to be explored further for actual risk analysis. It should be noted that curie inventories used in Release Fraction calculations are assumed values.

\section{Baseline Data from SAR and Dose Adjustment}

The SARs for three facilities were reviewed and thirteen discrete events for Facility \#1, six events for Facility \#2, and five events for Facility \#3 were selected. It should be noted that no rigorous evaluation was conducted for the event sequences in terms of mutual exclusiveness. It was simply assumed that these events are mutually exclusive (again, the purpose of the Example Case Analysis is to demonstrate the protocol, not actual risk estimation for these facilities). Those events that are more frequent than once a year are lumped into one event, but the consequences were added (see Footnote 4 of Table 1). The resulting baseline data are summarized in Table 1.

Some SARs present average individual doses at the site boundary and within 10 miles from the site boundary. However, these data are not readily available in many other SARs. In the Example Case Analysis, it is assumed that only MID (Maximum Individual Dose) values are available from SARs. The MID is based on an individual residing at the worst sector of the site boundary. Therefore, a following modification (denoted by $m_{1}$ ) due to differences in atmospheric dispersion factors $(\chi / Q)$ between the worst sector and average sector is needed:

$$
m_{1}=\frac{\sum_{i j}\left[(\chi / Q)_{i, j} \times P_{i, j}\right]}{(\chi / Q)_{M I D} \times \sum_{i, j}\left(P_{i j}\right)}
$$

where $P_{i, j}$ represents population in sector $i$ ( $i=1$ to 16), and distance $\mathrm{j}(\mathrm{j}=$ site boundary to 10 miles). Only one distance $(j=$ site boundary) is used for average site boundary dose (prompt fatality calculation). The calculated $m_{1}{ }^{\prime} s$ are 0.6 for the site boundary dose and 0.004 for the population dose for all three facilitics.

Since the SARs for these facilities do not present acute whole body doses, the acute dose is estimated from MID values. The MID in the SAR represents a 50-year cumulative effective dose equivalent (CEDE) and external doses are considered negligible compared to inhalation dose, since the dominant radionuclides, $\mathrm{Cs}-137, \mathrm{Pu}-238$, and $\mathrm{H}-3$ have negligible external dose factors. ${ }^{12}$ The CEDE values are converted to acute whole body dose ( 60 day or 0.17 year cumulative dose in this example to be consistent with $\mathrm{LD}_{50 / 60}$ ) with the following simplified modification factor $\left(m_{2}\right)$ based on effective half-life:

$m_{2}=\frac{1-\exp (-0.17 \Lambda)}{1-\exp (-50 \Lambda)}$

where

$\Lambda=\frac{\ln 2}{T_{1 / 2}}$, and $T_{1 / 2}$ is effective half-life in units of years.

The individual doses are dominated by beta-gamma fission product (primarily Cs-137) for Facility \#1, Pu238 for Facility \#2, and $\mathrm{H}-3$ for Facility $\# 3$, hence effective half-lives of only these three radionuclides are considered in this analysis. Since the radioactive half-lives for these nuclides are significantly longer than biological half-lives, the effective half-lives are essentially the same as the biological half-lives. The following biological half-lives are used for the calculation; ${ }^{13,14} 110$ days $(0.3 \mathrm{yr})$ for cesium, 20 years for plutonium and 10 days $(0.027 \mathrm{yr})$ for tritium. Hence, the $\mathrm{m}_{2}$ 's are 0.32 for Cesium (Facility $\# 1$ ), 0.007 for Plutonium (Facility \#2), and 0.99 for Tritium (Facility \#3). Therefore, the overall dose modification factors $\left(m_{1} m_{2}\right)$ for converting MID to acute dose are $0.2(0.6 \times 0.32)$ for Facility \#1, 0.004 $(0.6 \times 0.007)$ for Facility \#2, and $0.6(0.6 \times 0.99)$ for Facility \#3. The modification factor for converting MID to average individual dose (CEDE) within 10 miles of the site boundary is 0.004 for all facilities. The results are summarized in Table 2.

Based on Table 2, the exceedance probabilities, $\mathrm{P}(>\mathrm{D})$ for prompt fatality and cancer fatality are calculated and they are summarized in Tables 4 and 5. 


\section{Confinement Capability and Source Terms}

The exceedance probabilities, $P(>X)$ vs. $X$ for the three facilities in Table 3 are plotted in Figure 3. It is clear from the figure that Facility \#3 releases the highest fraction of its inventory for both normal operation and low frequency accidents, while Facility $\# 2$ releases the least. Furthermore, the exceedance probabilitier decrease very rapidly as release fractions increase for Facility \#2 for lower exceedance probability. This trend is consistent with the characteristics of the source terms and confinement barrier performance as compared below. Therefore, the Limit Lines based on $\mathrm{P}(>\mathrm{X})$ can be useful engineering indicators for confinement barriers.

\begin{tabular}{|c|c|c|}
\hline & $\begin{array}{l}\text { Source Terms } \\
\text { Dispersibility }\end{array}$ & $\begin{array}{l}\text { Confinement } \\
\text { Capability }\end{array}$ \\
\hline Facility \#1 & $\begin{array}{l}\text { Moderate } \\
\text { (Volatile liquid) }\end{array}$ & $\begin{array}{l}\text { Concrete Cells } \\
\text { HEPA filter }\end{array}$ \\
\hline Facility \#2 & $\begin{array}{l}\text { Low } \\
\text { (Solid powder) }\end{array}$ & $\begin{array}{l}\text { Canyon } \\
\text { Sand Filter }\end{array}$ \\
\hline Facility \#3 & $\begin{array}{l}\text { High } \\
\text { (Pressurized Gas) }\end{array}$ & $\begin{array}{l}\text { Cell } \\
\text { Stripper }\end{array}$ \\
\hline
\end{tabular}

Another observation to be made from the $P(>X)$ plot is that accident events included in the SAR for Facility \#3 cover entire spectrum of release categories, but the same can not be asserted for Facility \#1 or Facility \#2. Further event analysis is needed to cover high release-low probability end of the spectrum for Facility \#1. Otherwise, an analyst may have to use a linear extrapolation to get $P(>X)$ for total release (approximately $10^{-4} / y r$ in this case, which may be too high ). There is a similar need for further event analysis for Facility $\# 2$ to assure the validity of the steepness of that curve for lower exceedance probabilities. Hence, the $P(>X)$ plot is aiso a useful tool to identify opportunities for improvement in a SAR.

\section{Prompt Fatality}

The Prompt Fatality, $F_{p}$ is calculated from Figure 4 and Table 4 for the 3 facilities. It is apparent from this table and figure that the exceedance probability is dominated by Facility \#1 anu becomes 0 when the acute site boundary dose reaches 80 rems. Therefore, the risk of prompt fatality is 0 for both the case of step function dose response (Eq 8) and linear dose response function ( $\mathrm{Eq} 11)$, since the above acute dose is well below $\mathrm{LD}_{50 / 60}$ of about 500 rems $^{15}$ or $\mathrm{D}_{1}$ values of 150 rems in the reference 7 . In terms of mathematical formalism:

$F_{p}=P(>500)=0$

or

$F_{p}=(1 / 250) \cdot \int_{150}^{400} P(>D) \cdot d D=0$

The Prompt Fatality goal is about $5 \times 10^{-7} / \mathrm{yr}$ based on the US accidental fatality rate of about $5 \times 10^{-4}$ per year. $^{16}$

\section{Cancer Fatality}

It shoulc be noted that the Cancer Fatality Safety Goal is related to "risk... from operation" while the Prompt Fatality Safety Goal is related to "risk... from accidents". The definition of "operation" is not clearly established. It may be interpreted as normal eperation including anticipated operational eccurrences which may occur once in a lifetime of the facility (i.e., exceedance probability of on the order of $0.01 / y r$ ). Or it may be interpreted more conservatively to include all hypothetical accidents upto the maximum accident (i.e., inciuding $\mathrm{D}_{\max }=$ $1.6 \mathrm{rem})$. Both cases are calculated below. The latest publication of BEIR $V^{17}$ suggested a cancer rate of $K$ $=8 \times 10^{-4} / \mathrm{rem}$, which is used in the following calculation.

The Cancer Fatality, $F_{c}$ is calculated from Eq (13) and Figure 5. The solid line in the Figure 5 represents a total $\mathrm{P}(>\mathrm{D})$ for the three facilities. By linear extrapolation, the $D$ value corresponding to $P(>D)=$ $10^{-2} / \mathrm{yr}$ is $7.8 \times 10^{-4}$ rem. Hence, the Cancer Fatality risk due to normal operition including anticipated operational occurrences of the three facilities is:

$F_{c}=\left(8 \times 10^{-4}\right) \cdot \int_{0}^{7.8(-4)} P(>D) \cdot d D=3 \times 10^{-8} / y r$

For the conservative case of including the maximum accident, by a linear extrapolation of the Limit Line up to $D_{\max }=1.6 \mathrm{rem}, \mathrm{P}(>1.6)$ is $8.2 \times 10^{-6} / \mathrm{yr}$ (dotted TOTAL line in Figure 5). The resulting Cancer Fatality risk is:

$F_{c}=\left(8 \times 10^{-4}\right) \cdot \int_{0}^{1.6} P(>D) \cdot d D=9 \times 10^{-8} / y r$.

The cance fatality rate in the USA is about $2 \times$ $10^{-3} / \mathrm{yr}$ (Reference 18). The DOE Cancer Fatality Safety goal of $2 \times 10^{-6} / \mathrm{yr}$ is met for the three facilities, with a subsidntial margin. 


\section{EVALUATION OF RISK COMPARISON GUIDELINE}

The Westinghouse M\&O Nuclear Facility Safety Committee has proposed a guideline for radiological risk comparison. ${ }^{4}$ The guideline is meant for evaluating the acceptability of consequences of individual accident events (that is, the Limit Line is a Farmer plot rather than CCDF Limit Line). As discussed previously, if the density of accident frequency is not bunched together right at the Farmer's Limit Line, it can be treated as CCDF Limit Line. The risk comparison guideline is replotted in Figure 6. First, it is noted that at an exceedance probability of $5 \times 10^{-7} / \mathrm{yr}$ the CEDE is about 35 rems (and acute dose would be much less). Therefore, the DOE Prompt Fatality goal of $5 \times 10^{-7} / y t$ or less is clearly met.

From the Equation (13), Cancer Fatality risk is

$$
\begin{aligned}
F_{c} & =\left(8 \times 10^{-4}\right) \cdot \int_{0}^{D_{\max }} P(>D) \cdot d D \\
& =\left(8 \times 10^{-4}\right)\left\{\int_{0}^{0.01} P(>D) \cdot d D\right. \\
& \left.+\int_{0.01}^{0.5} P(>D) \cdot d D+\int_{0.5}^{25} P(>D) \cdot d D\right\} \\
& =\left(8 \times 10^{-4}\right)\left\{1.0 \times 10^{-2}+2.8 \times 10^{-2}+3.8 \times 10^{-3}\right\} \\
& =3.3 \times 10^{-5} / y r
\end{aligned}
$$

The above calculation is based on the assumption that an average person within 10 miles from the site boundary is exposed to the same level of radiation as the Maximum Individual at the site boundary. Therefore, the above fatality risk should be scaled down according to the ratio of $x / Q$ 's between the worst sector site boundary and the average location within 10 miles. The worst sector site boundary $x / Q$ is approximately $5 \times 10^{-6} \mathrm{sec} / \mathrm{m}^{3}$ and annual average $\chi / Q$ within 10 miles (12 to 16 miles from center of SRS is used for simplicity) is approximately $2 \times 10^{-8}$ $\mathrm{sec} / \mathrm{m}^{3}$ for the SRS. ${ }^{19}$ Hence, actual Cancer Fatality risk based on the M\&O Limit Line for the SRS facilities is:

$\left(3.3 \times 10^{-5}\right) \cdot \frac{2 \times 10^{-8}}{5 \times 10^{-6}}=1.3 \times 10^{-7} / \mathrm{yr}$

which is well below the DOE's safety goal of $2 \times 10^{-6}$ /yr by a factor of 15 .

It should also be noted from the $M \& O$ Limit Line that the primary contribution to total Cancer Fatality risk comes from those events whose frequency is greater than $10^{-2} / \mathrm{yr}$ (i.e., $91 \%$ from normal operation including anticipated operational occurrences), and the contribution from rare events is not significant (i.e., $9 \%$ for those events with frequency of $10^{-2}$ to $10^{-6}$ per year).

In summary, it can reasonably be assured that meeting the proposed $\mathrm{M} \& \mathrm{O}$ risk comparison guideline for individual accidents for SRS facilities satisfies the DOE's safety goal for cancer fatality, provided that the risk comparison guideline is a reasonable approximation of the CCDF Limit Line for the site.

\section{CONCLUDING REMARKS}

The mathematical formalism based on exceedance probability (CCDF) and the protocol of estab'ishing the exceedance probability resulted in Indicators that provide insights regarding confinement performance, source terms, and SAR adequacy. The results are directly comparable to the DOE quantitative Fatality Safety Goals. By virtue of integrating (Dose Response $x$ PDF) over the entire dose range and addition of Limit Lines of all facilities within a site, the resulting Risk Indicators represent "Total Risk" of a site.

As shown in the Example Case Analysis, existing SAR data with some modification can be used according to the above protocol and mathematical formalism. The results are consistent with the objectives of the method. The results can further reveal weakness in existing data. Since the Fatality Safety Goals are set at such a low level $\left(10^{-6}\right.$ to $10^{-7}$ per year range), accident events in a SAR need to cover down to this range (Facility \#1) or until a trend can be established (Facility \#2).

It should be emphasized that the dose-responses (prompt fatality, cancer fatality) used in the Example Case Analysis may be a gross simplification of what is presented in the respective references, and it was done so only to show how the mathematical formalism works. It was also assumed that the SAR data are appropriate for this type of risk analysis, 
although the SAR data may be on the conservative side (i.e., higher than "average" parameters) in general.

Finally, it is concluded that the general risk guidelines proposed for individual accidents, when treated as a CCDF, are consistent with the DOE Fatality Safety goals. This indicates not only the validity of these guidelines, but also the importance of these guidelines as complementary to the Safety Goals.

\section{ACKNOWLEDGMENT}

Portions of this manuscript are based on the paper presented at the Safety Analysis Workshop-1992 sponsored by the Energy Facility Contractor Group
(EFCXOG) held at Salt Lake City during August 11$13,1992$.

\section{REFERENCES}

1. Regulatory Guide 1.70 , Standard Format and Content SAR for Nuclear Power Plants (NRC, 1978)

2. DOE 5481.1B, Safety Analysis and Review System (DOE, 1986)

3. SEN-35-91, Nuclear Safety Policy (DOE, 1991)

4. Hallinan, Brown, Muhlestein and Yoder, Radiological Risk Comparison Guidelines, Proceedings of Safety Analysis Workshop, p 50 (EFCOG,1992)

5. Munera \& Yadigaroglu, Nucl. Technology, Vol 74, p 229 (1986)

6. Cox and Baybutt, Nucl. Technology, Vol. 57, p 320 (1982)

7. Langham (Ed.), Radiobiological Factors in Manned Space Flight, Publ. 1487, NAS/NRC (1967)

8. Kaplan \& Garrick, Risk Anal, Vol.1, p 11 (1981)

9. Farmer, Nucl. Safety, Vol.8, p 539 (1967)
10. Okerent, Nucl. Technology, Vol. 27, p 304 (1975)

11. WASH 1400, Reactor Safety Study (NRC, 1975)

12. DOE 5400.5, Radiation Protection of Public and Environment (DOE, 1990)

13. ICRP, Publ 30, Part 1, Limit for Intakes of Radionuclides by Workers (ICRP, 1979)

14. ICRP, Publ 30, Part 40, Limit for Intakes of Radionuclides by Workers (ICRP, 1988)

15. Cronkite \& Bond, US Armed Forces Medical Journal 9, p 313 (1958)

16. NUREG/CR-1916, A Risk Comparison (NRC, 1981)

17. Health Effect of Exposure to Low Level of Ionizing Radiation, BEIRV (NAS/NRC, 1990)

18. Cancer Facts and Figures (American Cancer Society, 1981)

19. WSRC-RP-91-188, Generic SAR Chapter 3, Site Characteristics (1991) 


\section{APPENDIX. INTEGRAL VS. SUM}

In this appendix, $\int P(>C) \cdot d C$ and $\Sigma\left(f_{i} C_{i}\right)$ are compared. As discussed in the text, $\Sigma\left(f_{i} X_{i}\right)$ can be an underestimation of "total risk" (Note: Some SARs present this sum as a total risk) due to unanalyzed events and not including low probability-high consequence events. The three facilities analyzed in the Example Case are compared for release fraction below. Similar comparison can be made for dose. The integration is based on limit lines of the Figure 3, and the sums are based on Release and Frequency data in Table 1.

$$
\int_{0}^{\max } \mathrm{P}(>\mathrm{X}) \cdot \mathrm{dX} \quad \sum_{\mathrm{i}} \mathrm{f}_{\mathrm{i}} \cdot \mathrm{X}_{\mathrm{i}}
$$

$\begin{array}{lll}\text { Facility \#1 } & 1.3(-5)^{*} & 7.5(-7) \\ & 2.1(-5)^{\dagger} & \end{array}$

Facility \#2 $\quad 1.8(-7) \quad 1.1(-7)$

Facility \#3 $\quad 1.1(-3) \quad 2.8(-4)$

Note: All units are (/yr)

* upper limit of integration $=0.003$

$\dagger$ upper limit of integration $=1.0$
As can be seen in the above comparison, a sum of selected events is significantly smaller than an integration of exceedance probability. The same is true for the case of:

$\int \mathrm{P}(>\mathrm{D}) \cdot \mathrm{dD}$ vs. $\Sigma \mathrm{f}_{\mathrm{i}} \cdot \mathrm{D}_{\mathrm{i}}(\mathrm{rem} / \mathrm{yr})$ 
Table 1. Summary of SAR Data

\begin{tabular}{llllll} 
Event\# & Description & $\begin{array}{l}\text { Freq } \\
(\text { /yr) }\end{array}$ & $\begin{array}{l}\text { Release } \\
\text { (Curies) }\end{array}$ & $\begin{array}{l}\text { Release } \\
\text { (Fraction) }\end{array}$ & $\begin{array}{l}\text { MID, } \\
\text { CEDE } \\
\text { (rem) }\end{array}$ \\
\cline { 1 - 4 } Facility \#1 & & & & & \\
1 & Earthquake & $2.0(-3)$ & $1.5(+3)$ & $3.0(-3)$ & 1.1 \\
2 & Tank Explosion & $1.9(-3)$ & $3.0(+2)$ & $6.0(-4)$ & $2.6(-1)$ \\
3 & Cell Explosion & $2.1(-6)$ & $3.0(+2)$ & $6.0(-4)$ & $2.6(-1)$ \\
4 & PR Explosion & $2.2(-5)$ & $7.8(+0)$ & $1.6(-5)$ & $3.8(-3)$ \\
5 & PP Fire & $1.8(-1)$ & $7.2(-1)$ & $1.4(-6)$ & $2.6(-4)$ \\
6 & SPC Fire & $1.1(-2)$ & $8.9(-3)$ & $1.8(-8)$ & $1.8(-6)$ \\
7 & Uncont Reactn & $1.5(-2)$ & $3.0(-2)$ & $6.0(-8)$ & $3.3(-4)$ \\
8 & Tank Overflow & $5.0(-2)$ & $3.5(-2)$ & $7.0(-8)$ & $3.0(-5)$ \\
9 & Tank Overflow & $5.0(-1)$ & $7.5(-2)$ & $1.5(-7)$ & $6.0(-5)$ \\
10 & Tank Leak & 1.0 & $5.0(-2)$ & $1.0(-7)$ & - \\
11 & Melter Explo & $8.8(-2)$ & $1.4(-3)$ & $2.8(-9)$ & $1.7(-8)$ \\
12 & Tornado & $2.1(-5)$ & $8.6(-3)$ & $1.7(-8)$ & $4.6(-9)$ \\
13 & Canister Ruptr & $2.0(-4)$ & $5.6(-4)$ & $1.1(-9)$ & $6.6(-6)$ \\
& & & & & \\
Facility \#2 & MBCD & $3.7(-3)$ & $3.5(-2)$ & $8.8(-7)$ & $1.5(-2)$ \\
1 & LBCD & $2.1(-1)$ & $1.7(-2)$ & $4.3(-7)$ & $7.6(-3)$ \\
2 & EQ & $1.7(-4)$ & $1.4(-2)$ & $3.5(-7)$ & $6.5(-3)$ \\
3 & MBC & $4.0(-2)$ & $3.5(-4)$ & $8.9(-9)$ & $1.5(-4)$ \\
4 & MBD & $4.0(-2)$ & $3.5(-4)$ & $8.8(-9)$ & $1.5(-4)$ \\
5 & Normal Op & 1.0 & $8.0(-4)$ & $2.0(-8)$ & - \\
6 & & & & & \\
& & & & & \\
Facility \#3 & Bed Fire & $2.4(-4)$ & $6.0(+6)$ & $5.0(-1)$ & $1.1(-3)$ \\
1 & Tank Fire & $3.8(-6)$ & $1.2(+6)$ & $1.0(-1)$ & $2.3(-1)$ \\
2 & Reservoir & $3.0(-2)$ & $3.0(-4)$ & $2.5(-3)$ & $5.7(-6)$ \\
3 & Diffuser & $5.0(-1)$ & $7.5(-1)$ & $6.3(-8)$ & $1.4(-7)$ \\
4 & Normal Op & 1.0 & $1.0(+3)$ & $8.3(-5)$ & $1.4(-6)$ \\
5 & & & & &
\end{tabular}

Note 1: $2.0(-3)$ means $2.0 \times 10^{-3}$

Note 2: $\chi / \mathrm{Q}$ for MID is $5.0(-6) \mathrm{sec} / \mathrm{m}^{3}$

Note 3: Total Inventory for Facility \#1 is assumed 5.0(+5) curies

Total Inventory for Facility $\# 2$ is assumed $4.0(+4)$ curies

Total Inventory for Facility \#3 is assumed $1.2(+7)$ curies

Note 4: SAR Release Data for events more frequent than once a year were annualized.

For example, 2 leaks a year with 10 curies per leak was considered as $1.0 / y r$

frequency with 20 curies release. 
Table A. Summary of Adjusted Dose Data

\begin{tabular}{|c|c|c|c|c|}
\hline Event \# & $\begin{array}{l}\text { Frequency } \\
(y \mathrm{yr})\end{array}$ & $\begin{array}{l}\text { Max Individual } \\
\text { CEDE (rem) }\end{array}$ & $\begin{array}{l}\text { Site Boundary } \\
\text { Acute (rem) }\end{array}$ & $\begin{array}{l}10 \text { mile Average } \\
\text { CEDE (rem) }\end{array}$ \\
\hline $\begin{array}{l}\text { Facility \#1 } \\
1 \\
2 \\
3 \\
4 \\
5 \\
6 \\
7 \\
8 \\
9 \\
10 \\
11 \\
12 \\
13 \\
*\end{array}$ & $\begin{array}{l}2.0(-3) \\
1.9(-3) \\
2.1(-6) \\
2.2(-5) \\
1.8(-1) \\
1.1(-2) \\
1.5(-2) \\
5.0(-2) \\
5.0(-1) \\
\text { Norm Op } \\
8.8(-2) \\
2.1(-5) \\
2.0(-4) \\
-\end{array}$ & $\begin{array}{l}1.1(+0) \\
2.6(-1) \\
2.6(-1) \\
3.8(-3) \\
2.6(-4) \\
1.8(-6) \\
3.3(-4) \\
3.0(-5) \\
6.0(-5) \\
- \\
1.7(-8) \\
4.6(-9) \\
6.6(-6) \\
4.0(+2)\end{array}$ & $\begin{array}{l}2.2(-1) \\
5.2(-2) \\
5.2(-2) \\
7.6(-4) \\
5.2(-5) \\
3.6(-7) \\
6.6(-5) \\
6.0(-6) \\
1.2(-5) \\
- \\
3.4(-9) \\
9.2(-10) \\
1.3(-6) \\
8.0(+1)\end{array}$ & $\begin{array}{l}4.4(-3) \\
1.0(-3) \\
1.0(-3) \\
1.5(-5) \\
1.0(-6) \\
7.2(-9) \\
1.3(-6) \\
1.2(-7) \\
2.4(-7) \\
- \\
6.8(-11) \\
1.8(-11) \\
2.6(-8) \\
1.6(+0)\end{array}$ \\
\hline $\begin{array}{l}\text { Facility \#2 } \\
1 \\
2 \\
3 \\
4 \\
5 \\
6 \\
*\end{array}$ & $\begin{array}{l}3.7(-3) \\
2.1(-1) \\
1.7(-4) \\
4.0(-2) \\
4.0(-2) \\
\text { Norm Op } \\
-\end{array}$ & $\begin{array}{l}1.5(-2) \\
7.6(-3) \\
6.5(-3) \\
1.5(-4) \\
1.5(-4) \\
- \\
1.8(+4)\end{array}$ & $\begin{array}{l}6.0(-5) \\
3.0(-5) \\
2.6(-5) \\
6.0(-7) \\
6.0(-7) \\
- \\
7.2(+1)\end{array}$ & $\begin{array}{l}6.0(-5) \\
3.0(-5) \\
2.6(-5) \\
6.0(-7) \\
6.0(-7) \\
- \\
7.2(+1)\end{array}$ \\
\hline $\begin{array}{l}\text { Facility \#3 } \\
1 \\
2 \\
3 \\
4 \\
5 \\
*\end{array}$ & $\begin{array}{l}2.4(-4) \\
3.8(-6) \\
3.0(-2) \\
5.0(-1) \\
\text { Norm Op } \\
-\end{array}$ & $\begin{array}{l}1.1(-3) \\
2.3(-1) \\
5.7(-6) \\
1.4(-7) \\
1.4(-6) \\
2.3(+0)\end{array}$ & $\begin{array}{l}6.6(-4) \\
1.4(-1) \\
3.4(-6) \\
8.4(-8) \\
8.4(-7) \\
1.4(+0)\end{array}$ & $\begin{array}{l}4.4(-6) \\
9.2(-4) \\
2.3(-8) \\
5.6(-10) \\
5.6(-9) \\
9.2(-3)\end{array}$ \\
\hline
\end{tabular}

Note: The last events (denoted by ${ }^{*}$ ) in each facility represent dose of $100 \%$ release ratioed from the maximum analyzed events. 
Table 3. Summary of $\mathbf{P}(>\mathrm{X})$ Calculation

\begin{tabular}{lll} 
Release Category & Events & $\mathrm{P}(>\mathrm{X})_{2}(\mathrm{yr})$ \\
\hline Facility \#1 & & \\
$\mathrm{X}=1.0$ & - & $2.0(-3)$ \\
$\mathrm{X}=3.0(-3)$ & 1 & $3.9(-3)$ \\
$\mathrm{X}=6.0(-4)$ & $1+2+3$ & $3.9(-3)$ \\
$\mathrm{X}=1.6(-5)$ & $1+2+3+4$ & $1.8(-1)$ \\
$\mathrm{X}=1.4(-6)$ & $1+2+3+4+5$ & $6.8(-1)$ \\
$\mathrm{X}=1.5(-7)$ & $1+2+3+4+5+9$ & 1.0 \\
$\mathrm{X}=1.0(-7)$ & $1+2+3+4+5+9+10$ & \\
Facility \#2 & & - \\
$X=1.0$ & & $3.7(-3)$ \\
$X=8.8(-7)$ & 1 & $2.1(-1)$ \\
$X=4.3(-7)$ & $1+2$ & $2.1(-1)$ \\
$X=3.5(-7)$ & $1+2+3$ & 1.0 \\
$X=2.0(-8)$ & $1+2+3+6$ & \\
& & \\
Facility \#3 & & - \\
$X=1.0$ & - & $2.4(-4)$ \\
$X=5.0(-1)$ & 1 & $2.4(-4)$ \\
$X=1.0(-1)$ & $1+2$ & $3.0(-2)$ \\
$X=2.5(-3)$ & $1+2+3$ & 1.0 \\
$X=8.3(-5)$ & $1+2+3+5$ &
\end{tabular}

Table 4. Summary of $P(>D)$ Calculation

(Site Boundary Acute Dose for Prompt Fatality)

Dose Category (rem) Events $\quad P(>D),(/ y r)$

$$
\begin{aligned}
& \text { Facility \#1 } \\
& D=\operatorname{Dmax}(80) \\
& D=2.2(-1) \\
& D=5.2(-2) \\
& D=7.6(-4) \\
& D=6.6(-5) \\
& D=5.2(-5) \\
& D=1.2(-5)
\end{aligned}
$$

Facility \#2

$\mathrm{D}=\mathrm{Dmax}(72)$

$\mathrm{D}=6.0(-5)$

$\mathrm{D}=3.0(-5)$

$\mathrm{D}=2.6(-5)$

$\mathrm{D}=6.0(-7)$

$\begin{array}{ll}- & - \\ 1 & 2.0(-3) \\ 1+2+3 & 3.9(-3) \\ 1+2+3+4 & 3.9(-3) \\ 1+2+3+4+7 & 1.9(-2) \\ 1+2+3+4+5+7 & 2.0(-1) \\ 1+2+3+4+5+7+9 & 7.5(-1)\end{array}$

Facility \#3

$\mathrm{D}=\mathrm{Dmax}(1.4)$

$\mathrm{D}=1.4(-1)$

$\mathrm{D}=6.6(-4)$

$\mathrm{D}=3.4(-6)$

$\mathrm{D}=1.0(-7)$

$i$

$1+2$

3.7(-3)

$1+2+3$

$2.1(-1)$

$1+2+3+4+5$

$2.1(-1)$

$2.9(-1)$

2

$1+2$

$1+2+3$

$1+2+3+5$
$3.8(-6)$

$2.4(-4)$

$3.0(-2)$

1.0 
Table 5. Summary of $P(>D)$ Calculation

(Average CEDE within 10 miles for Cancer Fatality)

Dose Category (rem)

Events

$\mathrm{P}(>\mathrm{D}),(/ \mathrm{yr})$

Facility \#1

$D=D \max (1.6)$

$\mathrm{D}=4.4(-3)$

$\mathrm{D}=1.0(-3)$

$\mathrm{D}=1.5(-5)$

$\mathrm{D}=1.3(-6)$

$\mathrm{D}=1.0(-6)$

$\mathrm{D}=2.4(-7)$

$\begin{array}{ll}-1 & 2.0(-3) \\ 1+2+3 & 3.9(-3) \\ 1+2+3+4 & 3.9(-3) \\ 1+2+3+4+7 & 1.9(-2) \\ 1+2+3+4+5+7 & 2.0(-1) \\ 1+2+3+4+5+7+9 & 7.5(-1)\end{array}$

\section{Facility \#2}

$\mathrm{D}=\mathrm{Dmax}(72)$

$\mathrm{D}=6.0(-5)$

$\mathrm{D}=3.0(-5)$

$\mathrm{D}=2.6(-5)$

$\mathrm{D}=6.0(-7)$

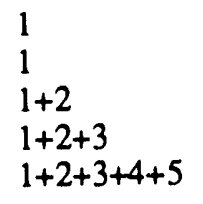

$3.7(-3)$

2.1(-1)

$2.1(-1)$

$2.9(-1)$

\section{Facility \#3 \\ $\mathrm{D}=\mathrm{Dmax}(0.0092)$ \\ $\mathrm{D}=9.2(-4)$ \\ $\mathrm{D}=4.4(-6)$ \\ $\mathrm{D}=2.3(-8)$ \\ $\mathrm{D}=5.6(-9)$}

$\begin{array}{ll}- & \\ 2 & 3.8(-6) \\ 1+2 & 2.4(-4) \\ 1+2+3 & 3.0(-2) \\ 1+2+3+5 & 1.0\end{array}$




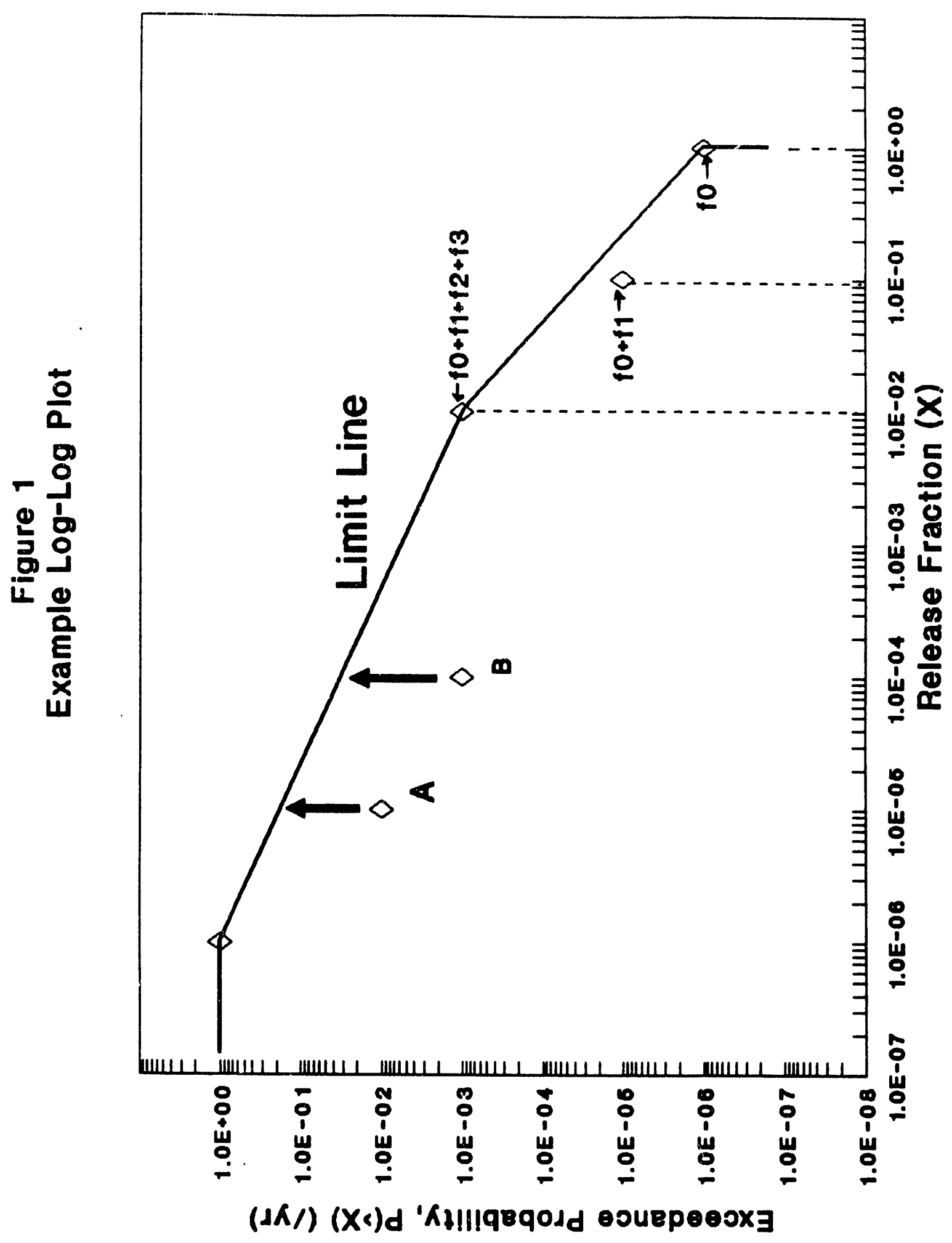

Figure 1. Example Log-log Plot 


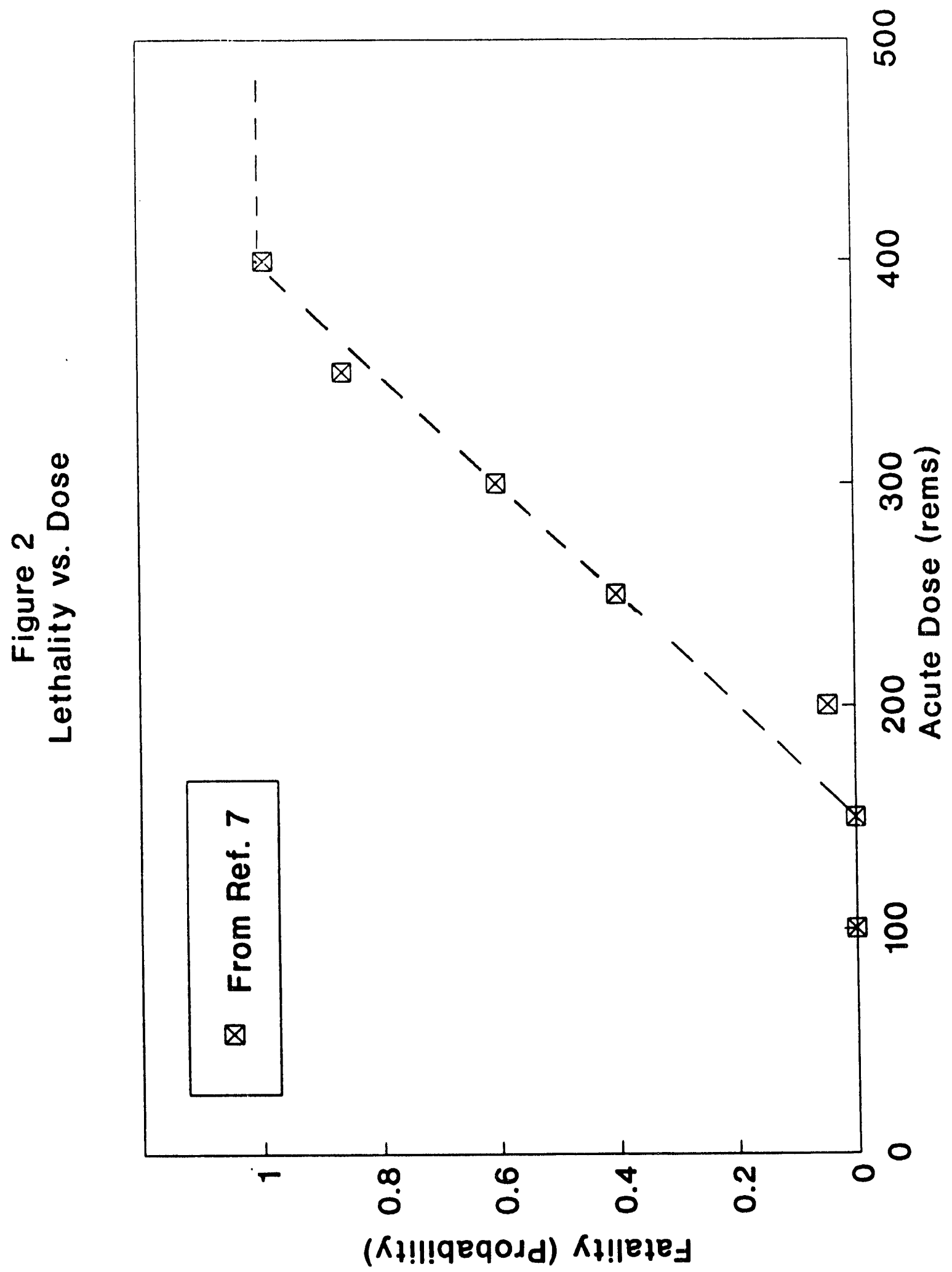

Figure 2. Lethality vs. Dose 


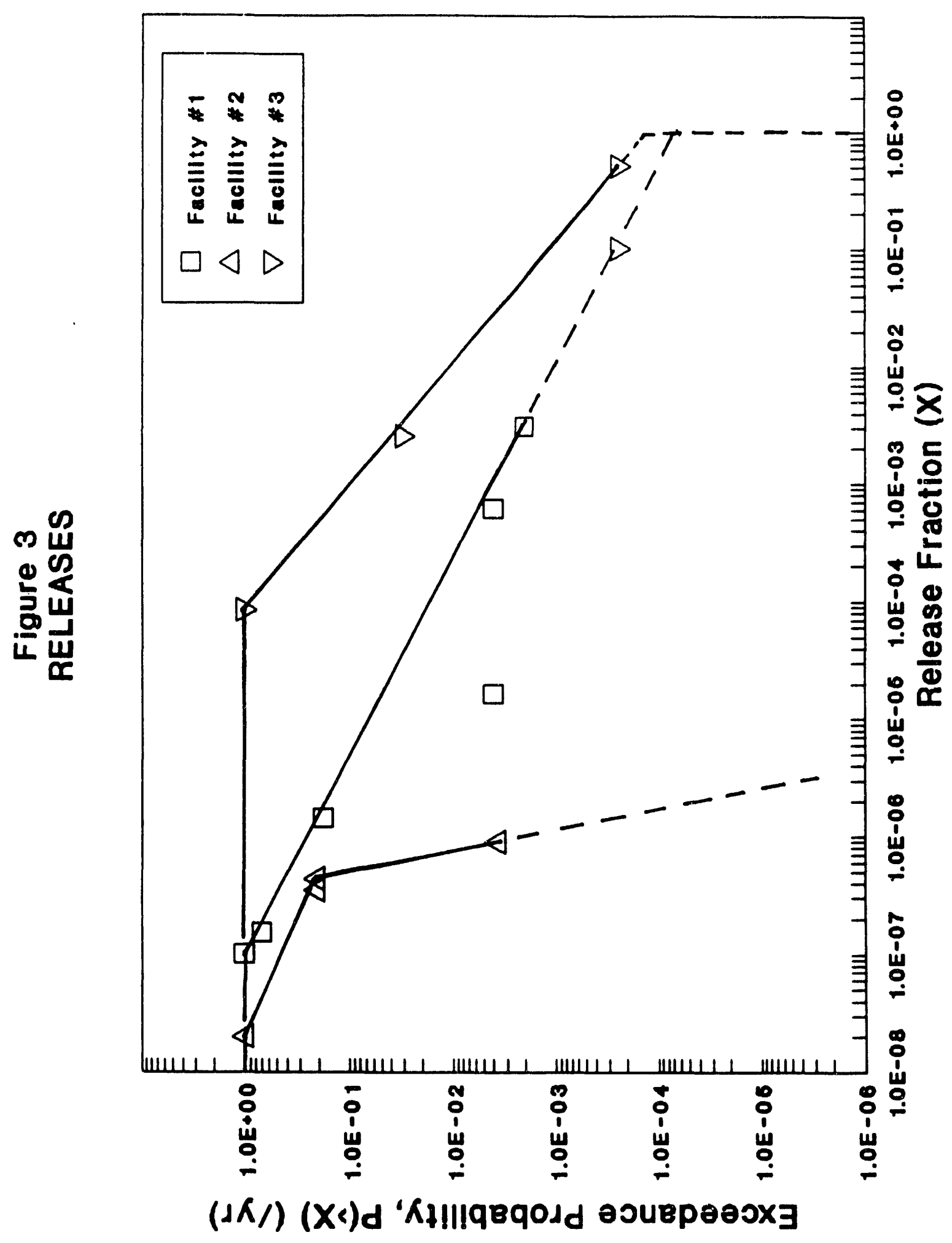

Figure 3. Releases 


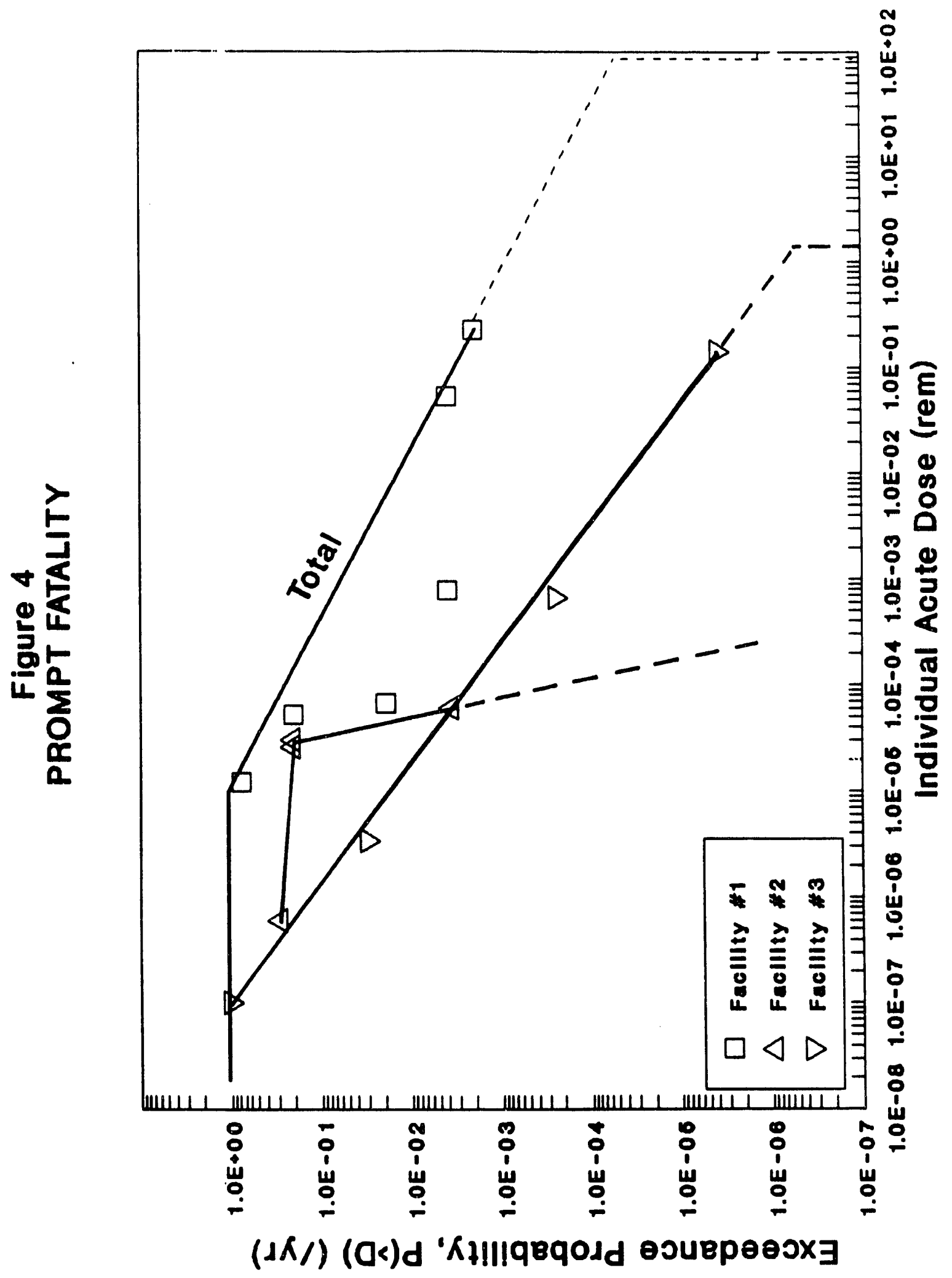

Figure 4. Prompt Fatality 


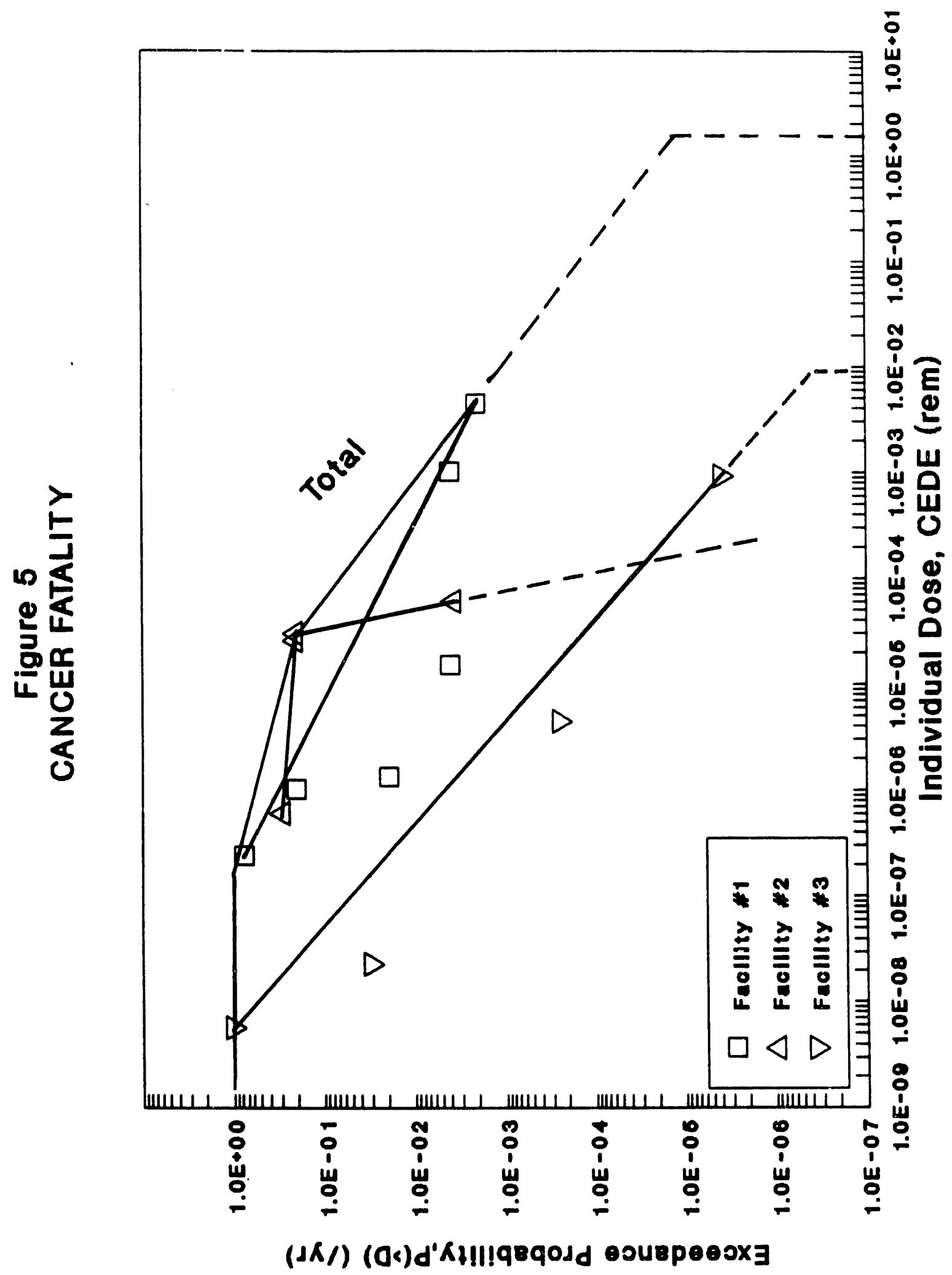

Figure 5. Cancer Fatality 


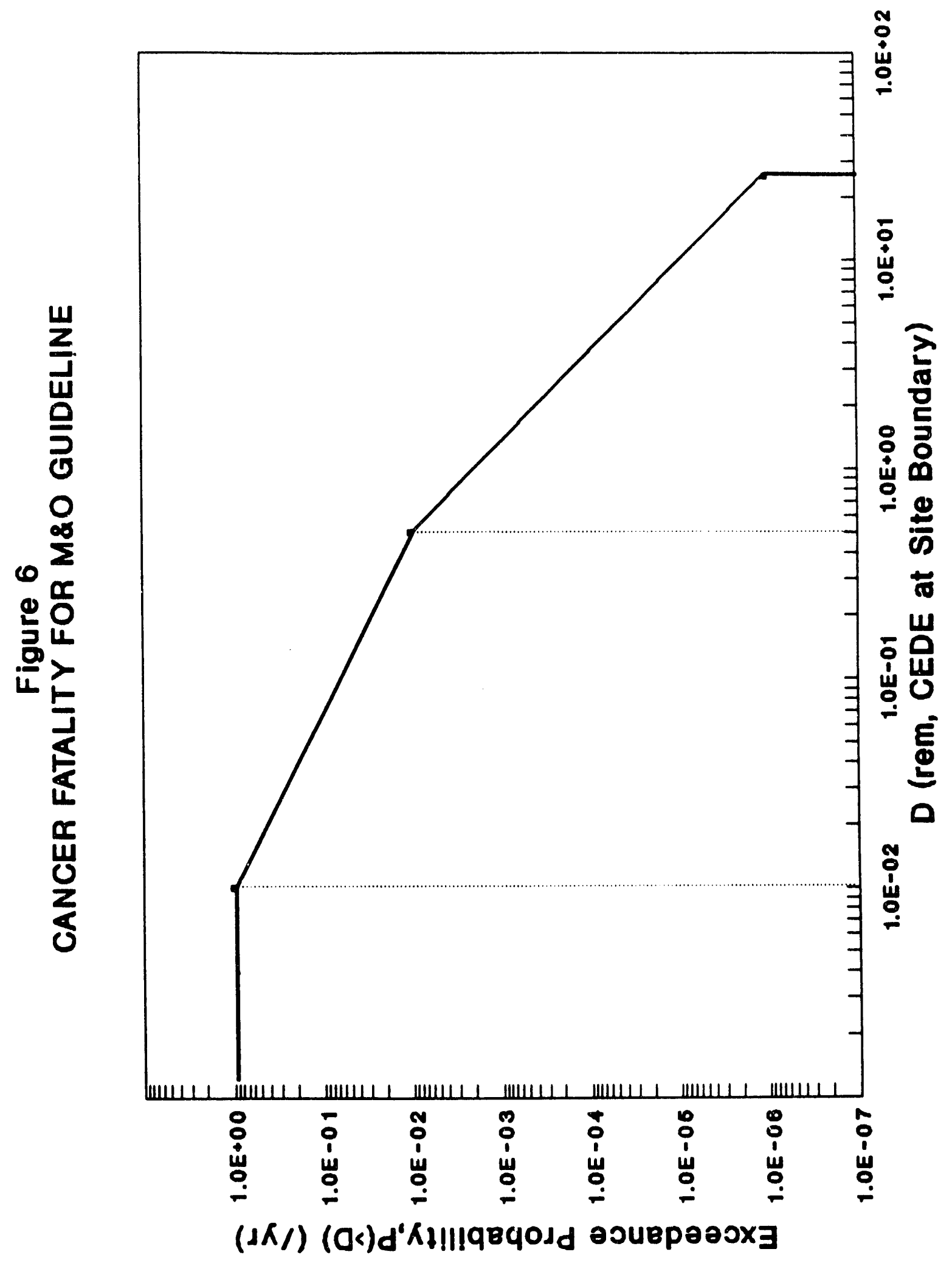

Figure 6. Cancer Fatality for M\&O Guideline 
BLANK 

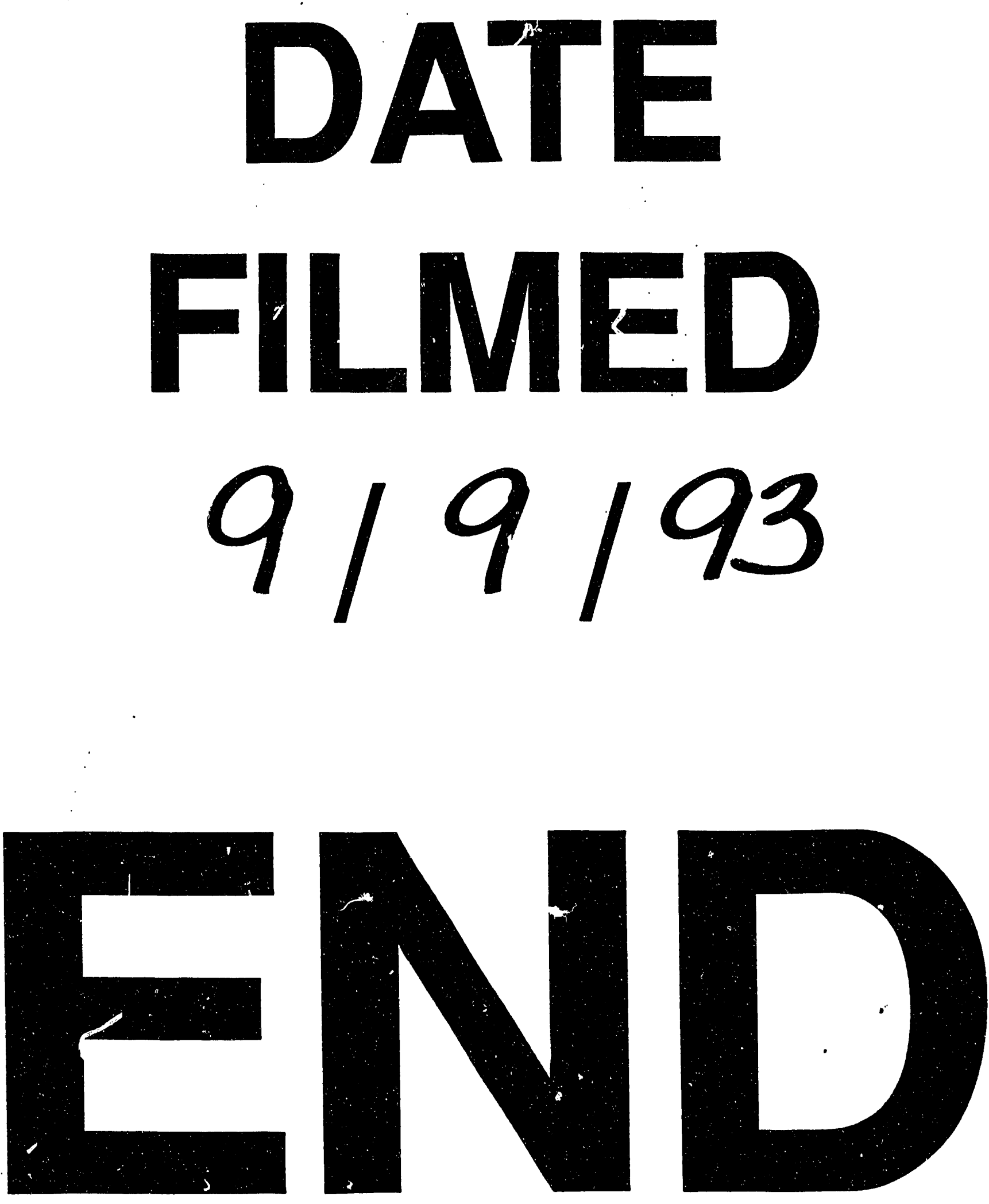
